\title{
A Mediastinal Mass Simulating an Aortic Dissection
}

Ali Laalou ${ }^{1 *}$, Abderrazak Chachi $^{1}$, Sara Jourani ${ }^{1}$, Mohammed El Jamili ${ }^{1}$, Dounia benzeroual ${ }^{1}$, Saloua El Karimi ${ }^{1}$, Mustapha El Hattaoui ${ }^{1}$

${ }^{1}$ Cardiology Department, Mohammed VI University Hospital, Marrakesh, Morocco

DOI: $10.36347 /$ sasjm.2021.v07i04.005

| Received: 17.03.2021 | Accepted: 23.04.2021 | Published: 29.04.2021

*Corresponding author: Ali Laalou

Abstract

Case Report

Described at the beginning of the 19th century by Shekleton, aortic dissection is a serious and life-threatening disease [1]. The diagnosis of dissection of the aorta is suspected by the presence of the classic triad: Severe abrupt chest pain, difference in upper extremity blood pressure and mediastinal widening on chest X ray. We report the case of 55 years old man with history of arterial hypertension presenting in the emergency department with the symptomatology of complicated aortic dissection. The diagnosis of mediastinal mass was retained.

Keywords: Aortic dissection, mediastinal mass.

Copyright $\odot 2021$ The Author(s): This is an open-access article distributed under the terms of the Creative Commons Attribution 4.0 International License (CC BY-NC 4.0) which permits unrestricted use, distribution, and reproduction in any medium for non-commercial use provided the original author and source are credited.

\section{INTRODUCTION}

Aortic dissection is a very serious condition, often fatal, revealed by several symptoms that may mimic those of other diseases, leading to delays in diagnosis.

The diagnosis of dissection of the aorta is suspected in the presence of the classic triad:

1. Severe abrupt onset, ripping or tearing Chest Pain that radiates to back AND

2. Pulse deficit or difference in upper extremity Blood Pressure $>20 \mathrm{mmHg}$ AND

3. Mediastinal Widening or aortic knob widening on Chest XRay (mediastinal enlargement, abnormality of the cardiac silhouette, pleural effusion)

$35 \%$ of aortic dissections nevertheless remain unsuspected in vivo, that's why several predictive clinical score for diagnosis of aortic dissection have been proposed and discussed by the authors.

\section{Case Report}

We report the case of Mr.BB, 55 years old, with history of arterial hypertension under amlodipine with poor adherence to medication, consulting the emergency department for intense chest pain, retrosternal, migrating, with inter-scapular radiation, evolving 1 week before his admission; the pain did not resolve under 1 st and 2 nd level of Nonsteroidal anti- inflammatory drugs (NSAIDs), and its intensity was increasing.

In addition, $\mathrm{Mr}$ B.B had functional impotence of the 2 lower limbs, of progressive installation, initially intermittent becoming permanent, without pain, sphincter disorders, or sensitivity disorders evolving 15 days before his admission.

The clinical examination found an apyretic patient, hemodynamically and respiratory stable. The cardiovascular examination found a blood pressure asymmetry (BP in the right upper limb $=180 / 100$ $\mathrm{mmHg}, \mathrm{BP}$ in the left upper limb $=100 / 60 \mathrm{mmHg}$ ), no cardiac murmur, neither carotid of abdominal aorta murmurs. The peripheral pulses are present and symetrical. No coldness of the lower extremities was found.

The patient presented a thoracic spinal syndrome, flaccid para pyramidal syndrome with hypotonia, abolished reflexes and negative Babinsky reflex bilaterally and symmetrically in the 2 lower limbs. In addition, we did not notice a sensory level, nor trophic disorders. The rest of the clinical examination: abdominal, pulmonary, osteoarticular and dermatological was without particularity.

Biological tests: complete blood count (CBC), erythrocyte sedimentation rate (ECR), blood sugar level, urea, ionogram, transaminases, troponin; were normal. ECG showed a sinus tachycardia, absence of 
conduction, repolarization or rhythmic disorders. The chest $\mathrm{x}$-ray found an enlargement of the upper mediastinum;

The thoracic-CT angiography was performed searching an aortic dissection. Contrariwise, we found a mediastinal, postero-superior mass, with bone lysis, vertebral compaction and endocanal infiltration.This mass included the primary carotid artery and the left subclavian artery, the arch of the aorta remained permeable.

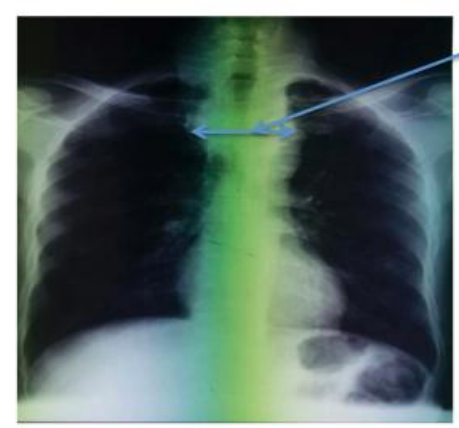

Image I: Chest $x$-ray Superior mediastinal enlargement, absence of cardiomegaly, no pulmonary parenchymal abnormalities, no bone abnormalities.

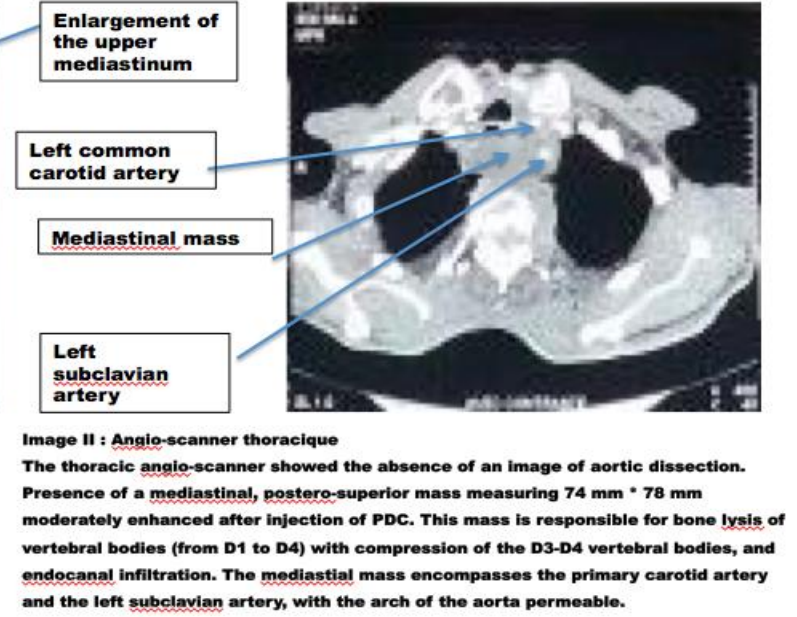

aortic dissection: 44495 patients between 1988 and 1996 presenting to the emergency department for chest pain or back pain were included in the study. After a statistical study, a clinical probability score including 3 clinical criteria was developed:

- Chest pain with an aortic origin was defined in the study as having an abrupt onset, with "ripping" nature

- Pulse asymmetry or blood pressure asymmetry

- Mediastinal enlargement.

In this study, in all patients $(100 \%)$ with the 3 clinical criteria, the diagnosis of aortic dissection was made (See Table I, II) [6].

In our patient, we found the 3 diagnostic criteria for aortic dissection: Aortic pain, pulse and blood pressure asymmetry and mediastinal enlargement. Several scores have been proposed in order to predict the clinical probability of an aortic dissection [2, 6, 7]. Von Kodolitsh [6] offers a predictive clinical score for

Table I, II: Calculation of the predictive score for aortic dissection according to 3 criteria (6)

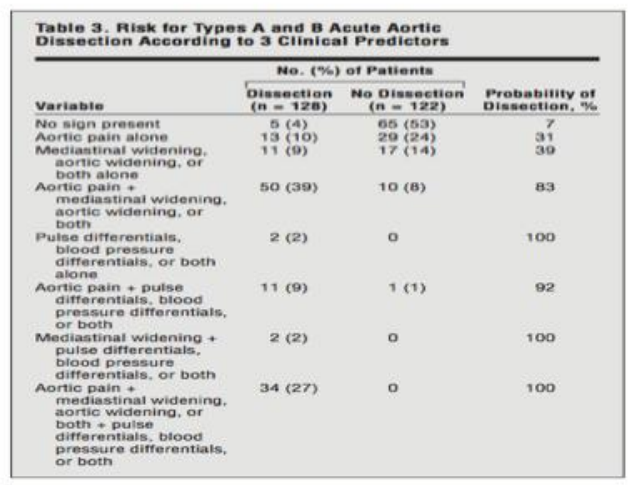

\begin{tabular}{|c|c|c|c|}
\hline Variable & $\begin{array}{l}\text { Nondissecting } \\
\text { Aortic Diseaset } \\
(n=21)\end{array}$ & $\begin{array}{l}\text { No Aortic } \\
\text { Oisease } \\
(n=36)\end{array}$ & $\boldsymbol{P}$ \\
\hline $\begin{array}{l}\text { Immediate onset of pain } \\
\text { alone }\end{array}$ & $8(38)$ & $25(69)$ & $<.05$ \\
\hline $\begin{array}{l}\text { Tearing or ripping pain } \\
\text { alone }\end{array}$ & $2(10)$ & 1 (3) & .27 \\
\hline $\begin{array}{l}\text { Immediate onset of pain } \\
\text { and tearing or ripping } \\
\text { pain }\end{array}$ & 1 (5) & $3(8)$ & .61 \\
\hline $\begin{array}{l}\text { Mediastinal widening. } \\
\text { aortic widening, or both }\end{array}$ & $17(81)$ & 10 (28) & $<.01$ \\
\hline $\begin{array}{l}\text { Murmur of aortic } \\
\text { regurgitation }\end{array}$ & $14(67)$ & $5(14)$ & $<.01$ \\
\hline $\begin{array}{l}\text { Pulse differentials, blood } \\
\text { pressure differentials, } \\
\text { or both and aortic pain }\end{array}$ & $1(5)$ & 0 & $\ldots$ \\
\hline
\end{tabular}


In addition, our patient presented on admission a functional impotence of abrupt onset with a para pyramidal syndrome in its flaccid phase on clinical examination.

Acute aortic dissection is one of the etiologies of acute paraplegia due to medullary infarction responsible for paraplegia. It is estimated that $10 \%$ of acute aortic dissections are associated to spinal cord syndrome [8]. The physiopathological mechanism mentioned is the obstruction of the intercostal segmental artery as well as the Adam-Kiewicz artery by the back and forth movements of the intimal flap of the false channel $[9,10]$.

In our patient, the motor deficit of the 2 lower limbs is explained by spinal compression by endo-canal infiltration of the mediastinal mass.

To sum up, in our patient:

- The mediastinal mass is responsible of the acute pain, probably due to compression of the neighboring organs.

- Tension asymmetry is caused by the mass encompassing the left subclavian artery.

- The visualization of the mediastinal mass is at the origin of the visualization of a superior mediastinal enlargement.

- Finally, paraplegia, is caused by bone lysis, and endo-canal infiltration of the mass with spinal compression and not by obstruction of the artery of Adam-Kiewicz.

\section{CONCLUSION}

We report in this observation, the case of a patient with a mediastinal mass with the full clinical presentation of an aortic dissection: History of hypertension, acute migrating chest pain, blood pressure asymmetry, functional impotence of the 2 lower limbs with brutal onset, mediastinal enlargement on chest XRay.

\section{REFERENCES}

1. Shekelton J. Healed dissecting aneurysm. Dublin Hosp Rep. 1922;3:231-232.

2. Klompas $\mathbf{M}$, Does this patient have an acute thoracic aortic dissection? JAMA. 2002 May 1;287(17):2262-72.

3. SpittelL PC, SpittelL Jr JA, Joyce JW, Tajik AJ, Edwards WD, Schaff HV, Stanson AW. Clinical features and differential diagnosis of aortic dissection: experience with 236 cases (1980 through 1990). InMayo Clinic Proceedings 1993 Jul 1 (Vol. 68, No. 7, pp. 642-651). Elsevier.

4. Slater EE, DeSanctis RW. The clinical recognition of dissecting aortic aneurysm, Am J Med. 1976 May 10;60(5):625-33.

5. Fann JI, Sarris GE, Mitchell RS, Shumway NE, Stinson EB, Oyer PE, Miller DC. Treatment of patients with aortic dissection presenting with peripheral vascular complications. Annals of surgery. 1990 Dec;212(6):705-13.

6. von Kodolitsch Y, Schwartz AG, Nienaber CA. Clinical prediction of acute aortic dissection. Archives of internal medicine. 2000 Oct 23;160(19):2977-82.

7. Rogers AM, Hermann LK, Booher AM, Nienaber CA, Williams DM, Kazerooni EA, Froehlich JB, O'Gara PT, Montgomery DG, Cooper JV, Harris KM. Sensitivity of the aortic dissection detection risk score, a novel guideline-based tool for identification of acute aortic dissection at initial presentation: results from the international registry of acute aortic dissection. Circulation. 2011 May 24;123(20):2213-8.

8. Khan IA, Nair CK. Clinical, diagnostic, and management perspectives of aortic dissection. Chest. 2002;122:311-2.

9. Le Bail B, Sartori E, Barrau Y, Herbreteau M. Paraplégie orthostatique secondaire à une dissection aortique. Revue neurologique (Paris). 2010;166(3):350-2.

10. Leys D, Cordonnier C, Masson C, Pruvo JP. Infarctus médullaires. In: Mas JL, Bousser MG, editors. Accidents vasculaires cérébraux. Paris: Doin; 2009. 1200 p. 8. 